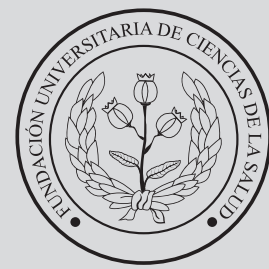

FUCS

\section{Re 作

Artículo de investigación

\title{
Relación entre estilos de aprendizaje y desempeño académico en un programa de instrumentación quirúrgica
}

\section{The relationship between learning styles and academic performance in a surgical instrumentation program}

Wanderley Augusto Arias ${ }^{a}$

Edgar Antonio Ibáñez

${ }^{a}$ Candidato a Magíster en Epidemiología Clínica. Programa de Instrumentación Quirúrgica, Grupo de Investigación Educación Superior en Salud. Facultad Escuela Colombiana de Medicina, Universidad El Bosque. Bogotá DC, Colombia.

${ }^{b}$ Magíster en Epidemiología Clínica. Candidato a Doctor en Estudios Politicos. Grupo de Investigación Medicina Comunitaria y Salud Colectiva, Facultad Escuela Colombiana de Medicina, Universidad El Bosque, Bogotá DC, Colombia.

\section{R E S U M E N}

Introducción: el presente estudio pretende determinar la relación entre los estilos de aprendizaje y el desempeño académico de los estudiantes de instrumentación quirúrgica de una institución de educación superior en 2019. Métodos: estudio de corte transversal analítico en 148 estudiantes de II a VII semestre de un programa de instrumentación quirúrgica en Bogotá, a quienes se aplicó la prueba CHAEA y se indagó sobre su desempeño académico para observar la relación entre estos. Resultados: el estilo predominante fue el reflexivo $41,9 \%(n=62)$ y el desempeño promedio $3,84 \pm 0,24$. No se encontró asociación entre estilos de aprendizaje y desempeño académico $(\mathrm{p}>0,05)$. Conclusión: no existe una relación estadísticamente significativa entre estilo de aprendizaje y desempeño académico, debido probablemente a homogeneidad entre los estudiantes.

Palabras clave: aprendizaje, educación superior, rendimiento académico, estilos de aprendizaje.

(C) 2020 Fundación Universitaria de Ciencias de la Salud - FUCS. Este es un artículo Open Access bajo la licencia CC BY-NC-ND (http://creativecommons.org/licenses/by-nc-nd/4.0/).

\section{INFORMACIÓN DEL ARTÍCULO}

Historia del artículo:

Fecha recibido: diciembre 6 de 2019 Fecha aceptado: mayo 21 de 2020

\footnotetext{
Autor para correspondencia. Wanderley Augusto Arias waarias@unbosque.edu.co
}

DOI

10.31260/RepertMedCir.01217372.1002 
Introduction: this study aims to determine the relationship between learning styles and academic performance of surgical instrumentation students in a higher education institution in 2019. Methods: analytical cross-sectional study of 148 students of semesters II to VII of a surgical instrumentation program in Bogotá. The CHAEA test was applied to these students and their academic performance was investigated to observe the relationship between them. Results: the predominant style was reflexive in $41.9 \%(\mathrm{n}=62)$ and the average performance was $3.84 \pm 0.24$. No association was found between learning styles and academic performance $(\mathrm{p}>0.05)$. Conclusion: there is no statistically significant relationship between learning style and academic performance probably due to homogeneity between students.

Key words: learning, higher education, academic performance, learning styles.

(C) 2020 Fundación Universitaria de Ciencias de la Salud - FUCS. This is an open access article under the CC BY-NC-ND license (http://creativecommons.org/licenses/by-nc-nd/4.0/).

\section{INTRODUCCIÓN}

En la actualidad educativa el análisis de los procesos de enseñanza-aprendizaje tomando como punto de partida modelos como el aprendizaje significativo juegan un papel fundamental las características de los estudiantes, pues conocer dichas cualidades permitirán al docente programar una estrategia de formación más efectiva. ${ }^{1}$ Entre estas características particulares están los denominados estilos de aprendizaje, los cuales según Alonso y Gallego (2006) son una serie de distintos comportamientos reunidos bajo una sola etiqueta que contribuyen a establecer lazos con la realidad, ayudando a clasificar y analizar los comportamientos. ${ }^{2}$

Uno de los procesos que mide la apropiación del conocimiento conceptual durante el pregrado es la valoración del desempeño o rendimiento académico, denominado por algunos autores como "evaluación educacional", en el cual cada asignatura o curso tiene una escala cuantitativa y cualitativa que permite identificar el nivel de apropiación del estudiante en cuanto a los conceptos que ha desarrollado en las asignaturas. En ciencias de la salud el proceso es más integrador y complejo, involucrando una visión holística del estudiante sobre los objetivos planteados en relación con el aprendizaje y las competencias determinadas en la estructura curricular. ${ }^{1,3}$

Dicho desempeño permitirá la promoción del aprendiz al nivel siguiente y por tanto juega un papel importante dentro del ejercicio académico del pregrado. Tal cuantificación se puede considerar como una variable dependiente puesto que se involucran muchos factores a la hora de generar la valoración y el proceso de cómo aprende el estudiante, así como su motivación, convirtiéndose en protagonistas al calificar mejor algunos estilos frente a otros. ${ }^{4}$
El presente estudio pretende determinar la relación entre los estilos de aprendizaje de los estudiantes de instrumentación quirúrgica de una institución de educación superior en Bogotá y su desempeño académico.

\section{Estilos de aprendizaje y la evaluación}

Siempre ha sido un verdadero reto identificar la forma como el ser humano aprende, comprendiendo este fenómeno como un engranaje psicológico o quizás biológico, donde confluyen una serie de factores, sustancias y procesos que tienen el único objetivo de permitir el almacenamiento efectivo de información en el individuo.

En las teorías aristotélicas como el empirismo y los fundamentos básicos de la epistemología y el aprendizaje, el individuo busca la forma de aprender y aprehender conceptos para enriquecer sus conocimientos. En palabras del mismo Aristóteles afirmaba que "todos los hombres poseen por naturaleza el deseo de saber", se comprende que el proceso de aprendizaje corresponde a una necesidad natural e innata del ser humano y que por ende todo el tiempo está en busca de ese saber. ${ }^{5}$

Con el advenimiento de nuevos conocimientos y el crecimiento acelerado de saberes, es natural que el estudiante genere nuevas estrategias y formas de aprender, siendo parte de la evolución. ${ }^{6}$ Es por esto que se han creado nuevas teorías acerca del aprendizaje y la consolidación del conocimiento, de modo que en la actualidad se relatan formas, enfoques y estilos de aprendizaje, así como estrategias para que al estudiante le sea más fácil realizar dicho proceso, denominadas metodologías de enseñanza. 


\section{MATERIALES Y MÉTODOS}

Se realizó un estudio de corte transversal analítico correlacional aplicado en una población de estudio representativa de 148 estudiantes de II a VII semestre, pertenecientes a una población marco de 272 estudiantes en un programa de instrumentación quirúrgica de una institución de educación superior en la ciudad de Bogotá. Como variables se tomaron el semestre cursado, edad, género, promedio acumulado y el estilo de aprendizaje. El promedio acumulado en la escala de 0 a 5 fue consultado en el instrumento (se dicotomizó la variable en dos características: menores de 4,0 y mayor o igual a 4) y para identificar el estilo de aprendizaje se utilizó la escala CHAEA validada al español, la cual fue diseñada para identificar estilos de aprendizaje dominantes. El instrumento consta de 80 aseveraciones que miden cuatro estilos de aprendizaje. Estos son activo, reflexivo, teórico y pragmático ${ }^{7}$, los estudiantes con predominio de dos o más estilos de aprendizaje se categorizaron como multimodales. La aplicación del instrumento se realizó de manera presencial, con un diligenciamiento voluntario por parte de los participantes, y su aplicación tomó cerca de 20 minutos. La tabulación se realizó mediante microsoft excel 365, en cuanto a la depuración y el procesamiento de datos se utilizó SPSS versión 25. Para el análisis bivariado se usó la prueba chi cuadrado de Pearson y el coeficiente de contingencia. El nivel de significancia fue $5 \%$.

\section{RESULTADOS}

Se encontró que la edad promedio fue 20,3 $\pm 2,4$ años, con un mínimo de 18 y máximo de 32 años y un coeficiente de variación de $11.82 \%$. El género predominante fue el femenino con 87,7\% $(\mathrm{n}=130)$. En lo relacionado con la distribución por estilos de aprendizaje, se encontró predominante el reflexivo $(n=62)$, mientras que la menor proporción fue para el activo $(\mathrm{n}=13)$ (figura 1).

Por otro lado, teniendo en cuenta el desempeño académico el promedio fue de 3,84 $\pm 0,24$ siendo el mínimo de 3,0 y el máximo de 4,4, con coeficiente de variación de $6.25 \%$. La mayoría de los estudiantes tuvieron un promedio menor de 4,0 representando el $81,1 \%(n=120)$ y los de mayor o igual a 4,0 fue 18,9\% $(\mathrm{n}=28)$. Al realizar el análisis de relación entre el estilo de aprendizaje y el desempeño académico se halló que el predominante fue el reflexivo con un promedio

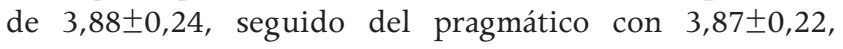

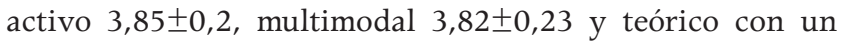
promedio de $3,8 \pm 0,33$; no se encontró variación entre los estilos de aprendizaje y el rendimiento (figura 2). Entre los estilos predominaron en todas las calificaciones $<4$, entre las calificaciones los estilos fueron similares (tabla 1). No se encontró asociación entre estilo de aprendizaje y desempeño académico $(\mathrm{p}=0,884)$ y el coeficiente de contingencia fue de 0,088 considerado como bajo.

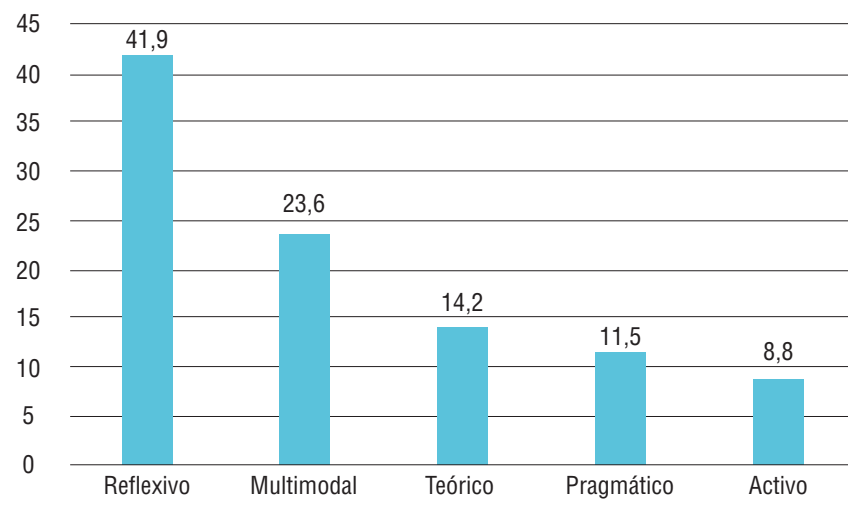

Figura 1. Distribución por estilo de aprendizaje.

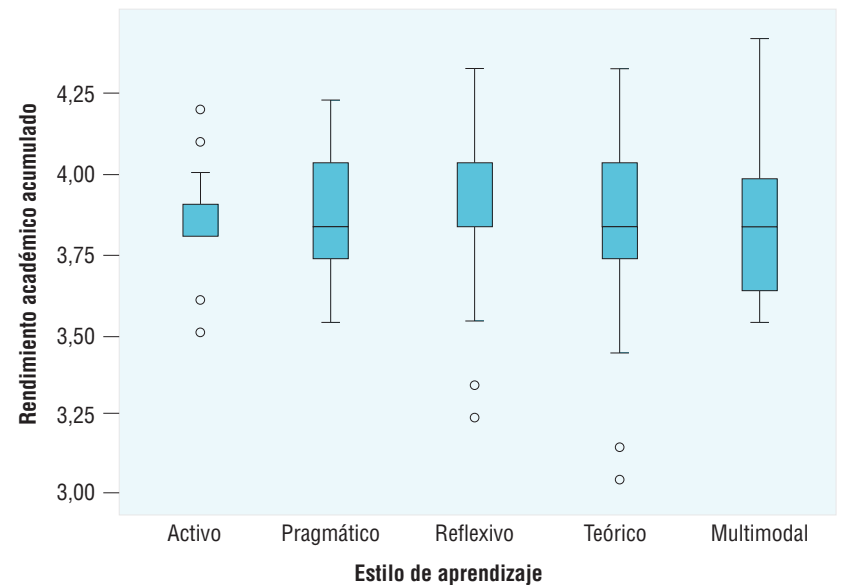

Figura 2. Diagrama de caja entre estilos de aprendizaje y desempeño acumulado.

Tabla 1. Asociación entre estilos de aprendizaje y desempeño de acuerdo con categoría y proporción

\begin{tabular}{|c|c|c|c|c|}
\hline & & & \multicolumn{2}{|c|}{ Rendimiento Académico } \\
\hline & & & $<4$ & $>0$ igual a 4 \\
\hline \multirow{5}{*}{$\begin{array}{l}\text { Estilo de } \\
\text { aprendizaje }\end{array}$} & Activo & $\begin{array}{c}n \\
\% \text { Fila } \\
\% \text { Columna }\end{array}$ & $\begin{array}{c}11 \\
84,60 \% \\
9,20 \%\end{array}$ & $\begin{array}{c}2 \\
15,40 \% \\
7,10 \%\end{array}$ \\
\hline & Pragmático & $\begin{array}{c}n \\
\% \text { Fila } \\
\% \text { Columna }\end{array}$ & $\begin{array}{c}13 \\
76,50 \% \\
10,80 \%\end{array}$ & $\begin{array}{c}4 \\
23,50 \% \\
14,30 \%\end{array}$ \\
\hline & Reflexivo & $\begin{array}{c}n \\
\% \text { Fila } \\
\% \text { Columna }\end{array}$ & $\begin{array}{c}50 \\
80,60 \% \\
41,70 \%\end{array}$ & $\begin{array}{c}12 \\
19,40 \% \\
42,90 \%\end{array}$ \\
\hline & Teórico & $\begin{array}{c}n \\
\% \text { Fila } \\
\% \text { Columna }\end{array}$ & $\begin{array}{c}16 \\
76,20 \% \\
13,30 \%\end{array}$ & $\begin{array}{c}5 \\
23,80 \% \\
17,90 \%\end{array}$ \\
\hline & Multimodal & $\begin{array}{c}n \\
\% \text { Fila } \\
\% \text { Columna }\end{array}$ & $\begin{array}{c}30 \\
85,70 \% \\
25,00 \%\end{array}$ & $\begin{array}{c}5 \\
14,30 \% \\
17,90 \%\end{array}$ \\
\hline
\end{tabular}




\section{DISCUSIÓN}

Una de las principales preocupaciones actuales en el entorno de la educación superior por parte de las instituciones de formación es la de brindar calidad, lo cual supondría la generación de un escenario académico donde cualquier estudiante pudiera tener no sólo los mejores docentes o instructores, o la mejor infraestructura, sino que se debería incluir el mejor acompañamiento y condiciones para que pueda aprender y tener buenos resultados académicos. Estos indicadores deberían ser primordiales a la hora de evaluar la calidad en la educación superior. ${ }^{8}$

De este modo, los resultados académicos pudieran ser uno de los indicadores para identificar si los esfuerzos mancomunados de la institución, educador y educando fueron exitosos, en el estudio se encontró que la distribución de los estudiantes en cuanto a su desempeño académico se presentó en mayor proporción en los niveles de bueno y excelente, lo cual permitiría inferir que la estrategia de los actores mencionados es de cierta manera efectiva, incluso teniendo en consideración las características particulares de cada estudiante, puesto que esta misma proporción se presentó en cada uno de los estilos de aprendizaje.

Así mismo, se encontró una relación que no es estadísticamente significativa entre un estilo de aprendizaje particular y el desempeño académico bueno o malo, lo cual contrasta con algunos estudios como el de García (2008) en el cual se evaluó la misma asociación en estudiantes de bachillerato, donde por medio de métodos inductivos y cualitativos se identificó una asociación entre el rendimiento académico y su estilo de aprendizaje; sin embargo el autor no presenta una correlación frente a las categorías de estilos y los valores de desempeño. Adicional a ello se pudiera objetar que el bachillerato corresponde a una población más diversa en relación con afinidades y que en la educación superior existe una homogenización de la educación. ${ }^{9}$

Sin embargo, evaluando los estudios realizados en talento humano en salud en distintas IES realizados por Esguerra G \& Guerrero P (2009) ${ }^{10}$ Nazar-Díaz D y col. (2014) ${ }^{11}$ y AltamiranoDroguett J y col. (2019) ${ }^{12}$ en donde se evaluaron estudiantes de psicología, dermatología y obstetricia respectivamente, se identificó un relacionamiento frente al desempeño académico y el estilo de aprendizaje, mostrando que en estas investigaciones el estilo de aprendizaje es un factor influyente en el desempeño académico de los estudiantes.

Por lo tanto, teniendo en cuenta los resultados de estos estudios se concluye que en áreas como la instrumentación quirúrgica, pareciera no existir una influencia significativa de los estilos de aprendizaje con el desempeño académico, donde el estilo de aprendizaje es independiente de las condiciones de los estudiantes.

Sin embargo, es relevante tener en cuenta variables que no se midieron y en posteriores estudios controlar la confusión residual, adicional a ello distinguir si características propias de la formación o de la IES son influyentes en la relación estilo de aprendizaje-desempeño académico y determinar la magnitud de su influencia. ${ }^{13}$

\section{FIN A N C I AC IÓ N}

Universidad El Bosque.

\section{DECLARACIÓN CONFLICTO DE INTERESES}

Los autores declaran no tener ningún conflicto de intereses.

\section{REFEREN CIAS}

1. Moreira MA. Aprendizaje significativo como un referente para la organización de la enseñanza. Archivos de Ciencias de la Educación 2017;11(12):e029. doi: https://doi.org/10.24215/23468866e029

2. Alonso Gacía CM, Gallego Gil DJ. Los estilos de aprendizaje; una propuesta pedagógica[Internet]. Chile: Universidad de Concepción; 2005 [citado 2009 octubre 1]; Recuperado de: https://educrea.cl/ wp-content/uploads/2015/04/Los-Estilos-de-Aprendizaje-UnaPropuesta-Pedagogica.pdf.

3. Pinilla Roa AE. Evaluación de competencias profesionales en salud. Rev Fac Med. 2013;61(1):53-70.

4. Acosta Muñoz JF, Cifuentes Mendivels RR. La condición motivacional en la evaluación educativa. Praxis \& Saber 2012;3(5):143-66. doi: https://doi.org/10.19053/22160159.1134

5. Betancourt D W. La filosofía como modo de saber Aristóteles, metafísica, A, 1 y 2, (980 a $21-983$ a 24). Praxis Filosófica 2013;37:29-55.

6. Bolívar López JM, Rojas Velásquez F. Estudio de la autopercepción y los estilos de aprendizaje como factores asociados al rendimiento académico en estudiantes universitarios. Revista De Educación a Distancia (RED). 2015;44:1-13.

7. Alonso CM, Gallego DJ, Honey P. Los estilos de aprendizaje. Procedimientos de diagnóstico y mejora. Bilbao, España: Ediciones Mensajero; 1995.

8. Camacaro PR. Evaluación de la calidad en educación superior. 2016. p. 21.

9. Estrada García A. Estilos de aprendizaje y rendimiento académico. bolredipe. 2018;7(7):218-28.

10. Esguerra Pérez G, Guerrero Ospina P. Estilos de aprendizaje y rendimiento académico en estudiantes de psicología. Divers Perspect Psicol. 2010;6(1):97-109.

11. Nazar-Díaz Mirón DM, Acevedo-Marrero Á, Sánchez-Valdivieso E. Estilos de aprendizaje y desempeño académico en dermatología. Dermatol Rev Mex 2014;58:157-64.

12. Altamirano-Droguett JE, Araya-Crisóstomo SP, Paz Contreras M. Estilos de aprendizaje y rendimiento académico de estudiantes de la carrera de obstetricia. Revista Ciencias de la Salud. 2019;17(2):276-92. doi: http://dx.doi.org/10.12804/revistas. urosario.edu.co/revsalud/a.7937

13. González de Alba V. Memoria 2010 de REICE. Revista Iberoamericana sobre Calidad, Eficacia y Cambio en Educación. 2011;9(1):121-7. 